\title{
Estimación del estado de carga y coeficientes de difusión en baterías de flujo redox
}

\author{
Alejandro Clemente, Andreu Cecilia i Ramon Costa-Castelló \\ Institut de Robòtica i Informàtica Industrial, CSIC-UPC, Llorens i Artigues 4-6, 08028 Barcelona \\ alejandro.clemente.leon@upc.edu, andreu.cecilia@upc.edu, ramon.costa@upc.edu
}

\begin{abstract}
Resumen
En este artículo se presenta el diseño de un observador de estado y parámetros para la estimación del estado de carga y algunos de los parámetros de difusión de una batería de flujo redox. El observador es de alta ganancia y se basa en un modelo dinámico no lineal, que utiliza un enfoque realista diferenciando entre las concentraciones totales de electrolito en las dos partes del sistema. Se muestra que el estado de carga se puede estimar mediante la medición del voltaje de celda y un observador de alta ganancia. No obstante, la precisión de la estimación se ve afectada por la incertidumbre en los coeficientes de difusión del sistema. Por esta razón, el observador se robustece mediante una estimación de parámetros adaptativos de inmersión e invariancia. Finalmente, los resultados se validan en una simulación numérica.
\end{abstract}

Palabras clave: Batería de flujo redox, estado de carga, observador no lineal adaptativo.

\section{INTRODUCCIÓN}

Es conocido que el uso de la energía renovable ha crecido durante los últimos años y seguirá aumentando en el futuro. La Agencia Internacional de Energía (AIE) ha estimado que en los próximos cinco años la capacidad total de energía renovable se expandirá en un 50\% [8], creciendo especialmente el número de plantas solares y eólicas. Uno de los principales inconvenientes de la energía renovable es su dependencia con las condiciones meteorológicas, dotándolas de un carácter intermitente, razón por la cual actualmente se trabaja en el desarrollo de almacenamiento de energía a gran escala como herramienta de apoyo. Es en este contexto, donde las baterías de flujo redox (BFR) se han convertido en una solución prometedora.

Una BFR es un sistema de almacenamiento de energía electroquímica que utiliza cuatro especies químicas divididas en dos subsistemas idénticos llamados anolito y catolito. Dispone de una celda electroquímica donde tienen lugar las reacciones redox durante un proceso de carga (corriente de catolito a analoito) o de descarga (del anolito al catolito). Por otro lado, dispone de dos tanques donde se almacena la energía y que están conectados a la celda a través de tuberías, que permiten la circulación de electrolito mediante dos bombas. En la Figura 1 se representa un esquema general de una BFR.

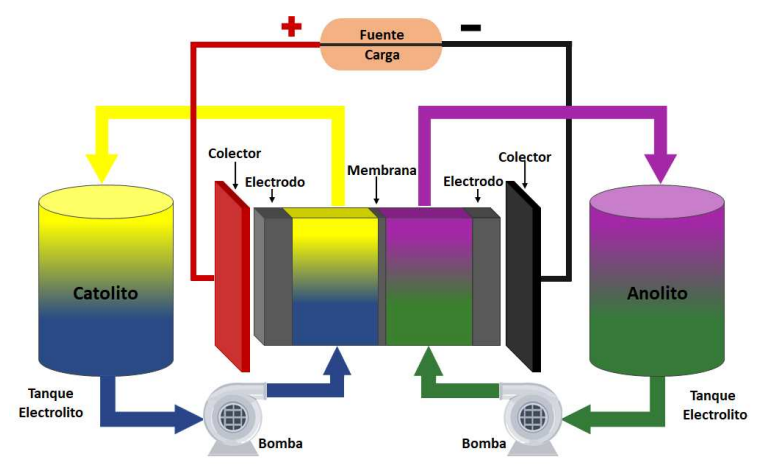

Figura 1: Esquema de una BFR con sus principales componentes.

Dado que la cantidad de energía almacenada es directamente proporcional al volumen de los tanques, las BFR son adecuadas para aplicaciones de energía a gran escala. Una de sus principales características es su modularidad entre la potencia y energía, la potencia se puede obtener mediante el diseño del stack en cuanto a medidas y número de celdas mientras que la energía viene exclusivamente determinada por el tamaño de los tanques. Como principal inconveniente, es que tienen una baja densidad energética, razón por la cual hace necesario disponer de grandes volúmenes de electrolito almacenado en tanques si se desea obtener una alta capacidad energética. No obstante, por su diseño flexible y otras características como son su larga duración o seguridad hacen de las BFR una buena elección como sistema de almacenamiento energético.

Entre los diferentes tipos de BFR, las baterías de flujo redox de vanadio (BFRV) se han convertido en la mejor opción debido al hecho de que todas las especies son óxidos de vanadio. De esta forma, no hay contaminación entre los dos electrolitos 
aunque exista una difusión de iones a través de la membrana. Una BFRV está compuesta por especies de vanadio en cuatro estados de oxidación diferentes. El anolito está compuesto por las especies $\mathrm{V}^{2+}$ y $\mathrm{V}^{3+}$, mientras que el catolito está compuesto por las especies $\mathrm{V}^{4+}$ y $\mathrm{V}^{5+}$. Dentro del estudio y análisis de este tipo de sistemas, uno de los desafíos más importantes es la determinación del estado de carga (SOC) que permite cuantificar la cantidad de energía almacenada en el sistema, permitiendo analizar el correcto funcionamiento de la batería [7].

El SOC se puede monitorizar directamente conociendo la concentración de especies dentro de los tanques, existiendo en la literatura múltiples técnicas destinadas a la estimación de dichas concentraciones como por ejemplo mediante el análisis del color, densidad o conductividad [12]. Sin embargo, estas técnicas son demasiado lentas, intrusivas e inexactas para ser una opción válida para el control en tiempo real. Teniendo en cuenta la imposibilidad de obtener una medida adecuada del SOC, algunos estudios proponen un observador para estimar su valor.

La medida más comúnmente utilizada para estimar el SOC es el voltaje en circuito abierto (OCV) que es la diferencia de potencial entre el catolito y anolito de la celda. Skyllas-Kazacos, que fue pionera en el uso de las BFRV, desarrolló un Filtro de Kalman Extendido (EKF) para estimar el SOC midiendo el OCV y asumiendo que la concentración de especies de vanadio tanto en la celda como en los tanques es la misma [16]. Sin embargo, el EKF se basa en la linealización del modelo, lo que da como resultado una solución local del problema de estimación y puede presentar poca robustez en otros puntos. Por esta razón, algunos autores implementaron el uso de observadores no lineales, como es el caso del observador de modo deslizante (SMO) [15], siendo especialmente útil para tratar con modelos no lineales.

Otra preocupación en la estimación del SOC es la incertidumbre paramétrica, que deteriora la precisión del observador. En consecuencia, algunos autores han estudiado el uso de técnicas de estimación adaptativa para reducir su efecto [13]. Desafortunadamente, las técnicas adaptativas disponibles se implementan comúnmente a través de métodos recursivos de mínimos cuadrados, por lo que la convergencia de la estimación de estados y parámetros solo se puede demostrar mediante la condición de excitación persistente.

Con todo ello, este artículo presenta un observador no lineal de alta ganancia [5] que puede estimar el SOC de un BFRV, tratando directamente con un modelo electroquímico no lineal más realista, que distingue entre los caudales de las dos partes del sistema, así como la concentración total de vanadio. Además, considera que no todos los coeficientes de difusión son conocidos, razón por la cual el observador está acoplado con un mecanismo de adaptación que estima los parámetros desconocidos [6]. La dinámica de adaptación se basa en ideas de inmersión e invariancia que permiten probar la convergencia de estados y parámetros aún en el caso de sistemas con relajada excitación persistente [1].

Este trabajo se ha organizado de la siguiente manera: La Sección 2 presenta el modelo no lineal así como las expresiones del OCV y del SOC. En la Sección 3, se presenta la formulación del problema. Un análisis de observabilidad es presentado en la sección 4 . La sección 5 presenta el observador de alta ganancia diseñado. La sección 6 presenta la dinámica de adaptación de parámetros. Todo el estudio ha sido validado mediante simulación numérica en la Sección 7. Finalmente, la Sección 8 presenta las conclusiones del trabajo.

\section{MODELO DINÁMICO DE LA BFRV}

Un modelo electroquímico dinámico que ha sido ampliamente utilizado es el propuesto por SkyllasKazacos que presenta la evolución de la concentración de especies dentro de la celda y los tanques [14]. Usando este enfoque, se ha formulado un modelo no lineal para una BFRV que tiene en cuenta que los caudales, $q$, y la concentración total de vanadio, $c_{v}$, son diferentes para el anolito y el catolito. Esta formulación no lineal se puede expresar en términos de las especies de vanadio $\mathrm{V}^{2+}$ y $\mathrm{V}^{5+}$ :

$$
\begin{aligned}
& \dot{x}_{1}=\frac{2}{v_{c}}\left[\left(x_{3}-x_{1}\right) q_{1}+\frac{I}{F}-k_{2} x_{1}-2 k_{5} x_{2}-k_{4} c_{v+}\right] \\
& \dot{x}_{2}=\frac{2}{v_{c}}\left[\left(x_{4}-x_{2}\right) q_{2}+\frac{I}{F}-k_{3} x_{1}-k_{5} x_{2}-k_{3} c_{v-}\right] \\
& \dot{x}_{3}=\frac{1}{v_{t}}\left(x_{1}-x_{3}\right) q_{1} \\
& \dot{x}_{4}=\frac{1}{v_{t}}\left(x_{2}-x_{4}\right) q_{2}
\end{aligned}
$$

donde $x_{1}$ es la concentración de $\mathrm{V}^{2+}$ en la celda del anolito, $x_{2}$ es la concentración de $\mathrm{V}^{5+}$ en la celda de catolito, $x_{3}$ es la concentración de $\mathrm{V}^{2+}$ en el tanque de anolito y $x_{4}$ la concentración de $\mathrm{V}^{5+}$ en el tanque de catolito. $q_{1}$ es el caudal en el anolito y $q_{2}$ en el catolito, $I$ es la corriente de carga o descarga, $F$ es la constante de Faraday, $k_{i}$ son los coeficientes de difusión de las especies de vanadio $\mathrm{V}^{i+}, v_{c}$ es el volumen de la celda y $v_{t}$ el volumen de cada tanque. 


\subsection{CÁlCULO DEL OCV}

En este trabajo, la ecuación del OCV considera el efecto de la concentración de protones dentro de la celda [11], así como las pérdidas óhmicas, y puede ser computado mediante la siguiente expresión:

$$
\begin{aligned}
E & =\frac{R T}{F} \ln \left[\left(\frac{x_{2} \cdot\left(c_{H_{+}}(0)+x_{2}\right)^{2}}{c_{v_{-}}-x_{2}}\right)\left(\frac{x_{1}}{c_{v_{+}}-x_{1}}\right)\right] \\
& +E^{\theta}+r I
\end{aligned}
$$

donde $E$ representa el voltaje de la celda OCV, $E^{\theta}$ es el potencial estándar del electrodo, $R$ es la constante del gas, $T$ es la temperatura de la celda, $c_{H_{+}}(0)$ es la concentración inicial de protones, $c_{v_{+}}$ es la concentración total de vanadio en el catolito, $c_{v_{-}}$en el anolito y $r$ es la resistencia óhmica.

\subsection{CÁLCULO DEL SOC}

Para calcular el SOC, en este trabajo se propone hacer una distinción entre la concentración de vanadio en el catolito y en el anolito, teniendo dos definiciones distintas:

$$
S O C_{-}=\frac{x_{3}}{c_{v_{-}}}, \quad S O C_{+}=\frac{x_{4}}{c_{v_{+}}} .
$$

Ambas definiciones se combinan para presentar una definición única del SOC del sistema completo, correspondiente al mínimo SOC de las dos partes:

$$
S O C=\min \left\{S O C_{-}, S O C_{+}\right\} .
$$

\section{FORMULACIÓN DEL PROBLEMA EN EL ESPACIO DE ESTADOS}

El objetivo es diseñar un observador que usando el voltaje de celda medido pueda estimar el valor de los estados $x_{3}$ y $x_{4}$, necesarios para la estimación del ECD mediante (3).

Para facilitar el diseño del observador, y sabiendo que la corriente y la temperatura se pueden medir fácilmente, es posible obtener una expresión más simplificada para trabajar. Suponiendo que $E^{\theta}$ y $r$ son constantes, se pueden restar del voltaje de la celda para obtener una expresión $h(\boldsymbol{x})$ que solo depende de las concentraciones de la celda $x_{1}, x_{2}$, $c_{H+}(0)$ y la concentración total de vanadio $c_{v_{-}} \mathrm{y}$ $c_{v_{+}}$:

$$
\begin{aligned}
h(\boldsymbol{x}) & =\frac{E-E^{\theta}-r I}{R T / F} \\
& =\ln \left[\left(\frac{x_{2} \cdot\left(c_{H_{+}}(0)+x_{2}\right)^{2}}{c_{v_{-}}-x_{2}}\right)\left(\frac{x_{1}}{c_{v_{+}}-x_{1}}\right)\right] .
\end{aligned}
$$

Teniendo en cuenta esta nueva expresión, es posible formular el sistema no lineal completo en el espacio de estados:

$$
\begin{aligned}
& \dot{\boldsymbol{x}}=\boldsymbol{A} \boldsymbol{x}+q_{1} \boldsymbol{H}_{1} \boldsymbol{x}+q_{2} \boldsymbol{H}_{2} \boldsymbol{x}+\boldsymbol{c I}+\boldsymbol{d} \\
& y=h(\boldsymbol{x})
\end{aligned}
$$

donde $\boldsymbol{x}$ son las concentraciones de especies de vanadio, y las matrices y vectores $\boldsymbol{A}, \boldsymbol{H}_{1}, \boldsymbol{H}_{2}, \boldsymbol{c}$ and $\boldsymbol{d}$ tienen los siguientes valores:

$$
\boldsymbol{A}=\frac{2}{v_{c}}\left[\begin{array}{cccc}
-k_{2} & -2 k_{5} & 0 & 0 \\
-k_{3} & -k_{5} & 0 & 0 \\
0 & 0 & 0 & 0 \\
0 & 0 & 0 & 0
\end{array}\right]
$$

$$
\boldsymbol{H}_{1}=\left[\begin{array}{cccc}
-\frac{2}{v_{c}} & 0 & \frac{2}{v_{c}} & 0 \\
0 & 0 & 0 & 0 \\
\frac{1}{v_{t}} & 0 & -\frac{1}{v_{t}} & 0 \\
0 & 0 & 0 & 0
\end{array}\right]
$$

$$
\boldsymbol{H}_{2}=\left[\begin{array}{cccc}
0 & 0 & 0 & 0 \\
0 & -\frac{2}{v_{c}} & 0 & \frac{2}{v_{c}} \\
0 & 0 & 0 & 0 \\
0 & \frac{1}{v_{t}} & 0 & -\frac{1}{v_{t}}
\end{array}\right]
$$

$$
c=\frac{2}{v_{c}}\left[\begin{array}{c}
1 / F \\
1 / F \\
0 \\
0
\end{array}\right] \quad \boldsymbol{d}=\frac{2}{v_{c}}\left[\begin{array}{c}
-k_{4} c_{v+} \\
-k_{3} c_{v-} \\
0 \\
0
\end{array}\right] \text {. }
$$

La identificación de los coeficientes de difusión sigue siendo un problema abierto e introducirá una incertidumbre significativa en la estimación del SOC, razón por la cual se robustecerá la estimación al acoplar al observador una dinámica de adaptación de parámetros.

\section{ANÁLISIS DE OBSERVABILIDAD}

El primer análisis que se debe realizar es la observabilidad del sistema presentado. Esto permite saber si los estados del sistema se pueden estimar a partir de la salida $y$.

En control, un sistema se considera (localmente) observable si satisface la condición de rango de observabilidad, lo que implica que el rango de un espacio de observabilidad $O_{s}$ debe ser igual al número de estados $n$ [4].

Para un sistema no lineal como el presentado para la BFRV, la condición de observabilidad se puede realizar de forma simple y automática calculando la codistribución de observabilidad $\Omega$. Para el caso 
de estudio, la codistribución de observabilidad de orden $4\left(\Omega_{4}\right)$ se calcula de la siguiente manera:

$$
\begin{aligned}
\Omega_{4} & =\operatorname{span}\{\nabla h\} \oplus \operatorname{span}\left\{\nabla L_{\boldsymbol{A} \boldsymbol{x}+q_{1} \boldsymbol{H}_{\boldsymbol{1}} \boldsymbol{x}+q_{2} \boldsymbol{H}_{2} \boldsymbol{x}} h(\boldsymbol{x})\right\} \\
& \oplus \operatorname{span}\left\{\nabla L_{\boldsymbol{A} \boldsymbol{x}+q_{1} \boldsymbol{H}_{1} \boldsymbol{x}+q_{2} \boldsymbol{H}_{2} \boldsymbol{x}}^{2} h\right\} \\
& \oplus \operatorname{span}\left\{\nabla L_{\boldsymbol{A} \boldsymbol{x}+q_{1} \boldsymbol{H}_{1} \boldsymbol{x}+q_{2} \boldsymbol{H}_{2} \boldsymbol{x}}^{3}\right\}
\end{aligned}
$$

donde span es el espacio generado, $\oplus$ es el operador de suma directa y $L^{i}$ es la función derivada de Lie que se puede calcular como:

$$
\begin{array}{r}
L_{\boldsymbol{f}(\boldsymbol{x})+\boldsymbol{g}(\boldsymbol{x})}^{1} h=\frac{\partial h}{\partial \boldsymbol{x}} \cdot(\boldsymbol{f}(\boldsymbol{x})+g(\boldsymbol{x})) \\
L_{\boldsymbol{f}(\boldsymbol{x})+g(\boldsymbol{x})}^{k} h=\frac{\partial L^{k-1} h}{\partial \boldsymbol{x}} \cdot(\boldsymbol{f}(\boldsymbol{x})+g(\boldsymbol{x})) .
\end{array}
$$

Llevando a cabo el análisis para el sistema formulado en (5) se puede demostrar que el rango de la codistribución de observabilidad $\Omega_{4}$ es máximo, por lo que el sistema es observable y las concentraciones se pueden estimar mediendo el voltaje de celda.

\section{DISEÑO DEL OBSERVADOR}

Una vez analizada la observabilidad del sistema, el siguiente paso corresponde al diseño de un observador apropiado capaz de estimar los estados $x_{3} \mathrm{y}$ $x_{4}$, que son los estados que determinan el SOC del sistema, y no aparecen implícitos en la expresión del OCV medido.

El procedimiento para diseñar el observador ha sido: primero, transformar el sistema a una forma canónica controlable, que acepta el diseño de un observador no lineal, y luego, invertir la transformación para recuperar la estimación en las coordenadas originales. En particular, se ha considerado el siguiente mapa:

$$
\Phi\left(\boldsymbol{x}, q_{1}, q_{2}\right) \triangleq\left[\begin{array}{c}
h(\boldsymbol{x}) \\
L_{\boldsymbol{A} \boldsymbol{x}+q_{1} \boldsymbol{H}_{1} \boldsymbol{x}+q_{2} \boldsymbol{H}_{2} \boldsymbol{x}} h(\boldsymbol{x}) \\
\vdots \\
L_{\boldsymbol{A} \boldsymbol{x}+q_{1} \boldsymbol{H}_{1} \boldsymbol{x}+q_{2} \boldsymbol{H}_{2} \boldsymbol{x}}^{3} h(\boldsymbol{x})
\end{array}\right] .
$$

Por medio de los resultados de observabilidad en la Sección 4, el mapa en (6) define un difeomorfismo entre las variables originales en el sistema en cuestión y las nuevas en la forma de retroalimentación estricta [3].

En consecuencia, es posible formular un observador de alta ganancia de la siguiente manera [2]:

$$
\begin{aligned}
\dot{\hat{\boldsymbol{x}}} & =\hat{\boldsymbol{A}} \hat{\boldsymbol{x}}+q_{1} \boldsymbol{H}_{1} \hat{\boldsymbol{x}}+q_{2} \boldsymbol{H}_{2} \hat{\boldsymbol{x}}+\boldsymbol{c} I+d \\
& +\left(\frac{\partial \Phi}{\partial \boldsymbol{x}}\right)^{-1}\left[\frac{\alpha_{1}}{\epsilon}, \frac{\alpha_{2}}{\epsilon^{2}}, \frac{\alpha_{3}}{\epsilon^{3}}, \frac{\alpha_{4}}{\epsilon^{4}}\right]^{\top}(y-h(\hat{\boldsymbol{x}}))
\end{aligned}
$$

donde $\alpha_{i}$ y $\epsilon$ son los parámetros del observador a diseñar y $\hat{\mathbf{A}}$ es la matriz A con los valores estimados de los parámetros desconocidos.

Lema 5.1 [10] Considere el observador de alta ganancia (7) con los parámetros $\alpha_{i}$ sintonizados de manera que el polinomio:

$$
s^{4}+\alpha_{1} s^{3}+\alpha_{2} s^{2}+\alpha_{3} s+\alpha_{4}
$$

sea Hurtwitz, con todas las raices en el semiplano izquierdo. Entonces, hay una constante positiva $\varepsilon^{*}$, tal que, para todo $\varepsilon \leq \min \left\{\varepsilon^{*}, 1\right\}$, el error de estimación del observador (7) se limita en última instancia de la siguiente manera

$$
\|\boldsymbol{x}-\hat{\boldsymbol{x}}\| \leq \kappa_{1}\|\boldsymbol{K}-\hat{\boldsymbol{K}}\|,
$$

donde $\kappa_{1}$ está formada por constantes positivas $y$ $\boldsymbol{K}=\left[\begin{array}{l}k_{2} \\ k_{5}\end{array}\right]$.

Observación 5.1 Para diseñar el $\epsilon$ es importante tener en cuenta sus propiedades. Por un lado, los valores pequeños de $\epsilon$ aseguran una convergencia rápida y robusta del observador. Por otro lado, valores demasiado pequeños inducirán el fenómeno de pico y agravarán la sensibilidad al ruido del observador [10]. Teniendo en cuenta estas cuestiones, es importante diseñar correctamente el valor de $\epsilon$ para evitar estos fenómenos, al tiempo que se obtiene una estimación correcta.

\section{DISEÑO DEL ESTIMADOR DE PARÁMETROS}

En este trabajo, para facilitar el estudio, se ha considerado que solo se desconocen los coeficientes de difusión $k_{2}$ y $k_{5}$. Se puede observar que dichos parámetros aparecen en la primera derivada de la función de salida (4). Por lo tanto, los parámetros desconocidos aparecen en el mapa de observabilidad considerado (6). En consecuencia, el observador siempre presentará un sesgo que no podrá reducirse aumentando su ganancia.

Teniendo en cuenta este problema, se propone acoplar al observador con un algoritmo de estimación de parámetros. Con este enfoque, una técnica común es estimar los parámetros del modelo a través de una dinámica de adaptación similar al descenso de gradientes. Esta estrategia permite un desacoplo entre los estados y la estimación de parámetros, mediante la cancelación de los parámetros desconocidos de la función de Lyapunov [17]. Sin embargo, la convergencia de los parámetros sólo puede demostrarse bajo la condición de persistente excitación la cual es muy restrictiva [17]. Como las BFRV normalmente 
operan alrededor de un punto de equilibrio, la condición de excitación persistente no se cumplirá genéricamente. En consecuencia, las técnicas similares al descenso de gradientes no pueden garantizar la convergencia de los parámetros.

Estos problemas pueden solucionarse mediante un método de estimación de parámetros basado en la técnica de Immersión e Invariancia (I\&I) [1]. Este enfoque se basa en el diseño de una variedad invariante y atractiva que se puede utilizar para estimar los parámetros.

Lema 6.1 Considere la siguiente dinámica de estimación de parámetros:

$$
\begin{aligned}
\dot{\hat{\theta}} & =-\frac{\partial \beta(\hat{\boldsymbol{x}})}{\partial \boldsymbol{x}}[f(\hat{\boldsymbol{x}}, \boldsymbol{u})+\varphi(\hat{\boldsymbol{x}})(\hat{\theta}+\beta(\hat{\boldsymbol{x}}))] \\
\hat{\boldsymbol{K}} & =\hat{\theta}+\beta(\hat{\boldsymbol{x}}),
\end{aligned}
$$

donde $f(\hat{\boldsymbol{x}}, u), \varphi(\hat{\boldsymbol{x}})$ y $\beta(\hat{\boldsymbol{x}})$ son los vectores de funciones

$$
\begin{gathered}
f(\hat{\boldsymbol{x}}, \boldsymbol{u})=\left[\begin{array}{c}
\frac{2}{v_{c}}\left[\left(\hat{x}_{3}-\hat{x}_{1}\right) q_{1}+\frac{I}{F}-k_{4} \hat{x}_{2}\right] \\
\frac{2}{v_{c}}\left[\left(\hat{x}_{4}-\hat{x}_{2}\right) q_{2}+\frac{I}{F}-k_{3} \hat{x}_{1}-k_{3} c_{v_{-}}\right]
\end{array}\right] \\
\varphi(\hat{\boldsymbol{x}})=\left[\begin{array}{cc}
-\hat{x}_{1} & -2 c_{v_{+}} \\
0 & -\hat{x}_{2}
\end{array}\right] \\
\beta(\hat{\boldsymbol{x}})=\frac{\gamma}{2}\left[\begin{array}{c}
-\frac{1}{2} \hat{x}_{1}^{2} \\
-\frac{1}{2} \hat{x}_{2}^{2}
\end{array}\right]
\end{gathered}
$$

siendo $\gamma$ una constante positiva de diseño.

Entonces, el error del estimador de parámetros convergirá hacia:

$$
\|\boldsymbol{K}-\hat{\boldsymbol{K}}\| \leq \kappa_{2}\|\boldsymbol{x}-\hat{\boldsymbol{x}}\|
$$

siendo $\hat{\boldsymbol{K}}=\left[\begin{array}{l}\hat{k}_{2} \\ \hat{k}_{5}\end{array}\right]$ y $\kappa_{2}$ una constante positiva.

Demostración 6.1 Considerando (11) se obtiene la siguiente expresión:

$$
\frac{\partial \beta(\hat{\boldsymbol{x}})}{\partial \boldsymbol{x}} \varphi(\hat{\boldsymbol{x}})=\gamma\left[\begin{array}{cc}
\frac{\hat{x}_{1}^{2}}{2} & \hat{x}_{1} c_{v_{+}} \\
0 & \frac{\hat{x}_{2}^{2}}{2}
\end{array}\right]
$$

que tiene valores singulares positivos.

Definiendo el siguiente variante $\boldsymbol{z}$ :

$$
\boldsymbol{z} \triangleq \hat{\theta}-\boldsymbol{K}+\beta(\boldsymbol{x})
$$

su dinámica $\boldsymbol{z}$ viene dada por:

$$
\begin{aligned}
\dot{\boldsymbol{z}} & =-\frac{\partial \beta(\boldsymbol{x})}{\partial \boldsymbol{x}} \varphi(\boldsymbol{x}) \boldsymbol{z}+\delta \\
& =-\gamma\left[\begin{array}{cc}
\frac{x_{1}^{2}}{2} & x_{1} c_{v_{+}} \\
0 & \frac{x_{2}^{2}}{2} .
\end{array}\right] \boldsymbol{z}+\delta
\end{aligned}
$$

donde $\delta$ se define como:

$$
\begin{aligned}
\delta & \triangleq \frac{\partial \beta(\boldsymbol{x})}{\partial \boldsymbol{x}}[f(\boldsymbol{x}, \boldsymbol{u})+\varphi(\boldsymbol{x})(\hat{\theta}+\beta(\boldsymbol{x}))] \\
& -\frac{\partial \beta(\hat{\boldsymbol{x}})}{\partial \boldsymbol{x}}[f(\hat{\boldsymbol{x}}, \boldsymbol{u})+\varphi(\hat{\boldsymbol{x}})(\hat{\theta}+\beta(\hat{\boldsymbol{x}}))] .
\end{aligned}
$$

Como las funciones $\beta, f(\boldsymbol{x}, \boldsymbol{u})$ and $\varphi(\boldsymbol{x})$ son (localmente) Lipschitz y el factor $\frac{\partial \beta(\boldsymbol{x})}{\partial \boldsymbol{x}}$ tiene un límite superior, es posible encontrar una constante positiva $L_{\delta}$ tal que:

$$
\|\delta\| \leq L_{\delta}\|\boldsymbol{x}-\hat{\boldsymbol{x}}\|
$$

Si se considera la siguiente función de Lyapunov como candidata

$$
V=\frac{1}{2} \boldsymbol{z}^{\top} \boldsymbol{z}
$$

su derivada es

$$
\dot{V}=-\gamma \boldsymbol{z}^{\top}\left[\begin{array}{cc}
\frac{x_{1}^{2}}{2} & x_{1} c_{v_{+}} \\
0 & \frac{x_{2}^{2}}{2} .
\end{array}\right] \boldsymbol{z}+\boldsymbol{z} \delta,
$$

y considerando que $\delta$ está limitada (16) se cumple la siguiente inecuación:

$$
\dot{V} \leq-\gamma \boldsymbol{z}^{\top}\left[\begin{array}{cc}
\frac{x_{1}^{2}}{2} & x_{1} c_{v_{+}} \\
0 & \frac{x_{2}^{2}}{2}
\end{array}\right] \boldsymbol{z}+\boldsymbol{z} L_{\delta}\|\boldsymbol{x}-\hat{\boldsymbol{x}}\| .
$$

Como la matriz no es $L_{2}$ integrable en toda la región operativa, es posible demostrar que hay una constante positiva $\kappa_{3}$ tal que la variable fuera de la variedad está delimitada en última instancia como

$$
\|\boldsymbol{z}\| \leq \kappa_{3}\|\boldsymbol{x}-\hat{\boldsymbol{x}}\|
$$

Finalmente, considerando la estimación del parámetro (10), la definición de coordenadas fuera de la variedad (13) y el hecho de que $\boldsymbol{\beta}(\mathbf{x})$ es Lipschitz, el límite (12) se puede deducir.

\subsection{ESTABILIDAD DEL OBSERVADOR}

Es importante seleccionar adecuadamente las condiciones en las que el acoplamiento entre el observador de estados y el estimador de parámetros crea una estructura estable.

Se ha demostrado que el error de estimación de estados converge a un valor que depende del error de estimación de parámetros, ver (9). Además, se cumple una propiedad similar para el error de estimación de parámetros, considerando el error de estimación de estado como una entrada, ver (12).

Lema 6.2 El acoplamiento entre el observador de alta ganancia (7) y la dinámica de adaptación (10) es estable si se cumple lo siguiente:

$$
\kappa_{1} \kappa_{2} \leq 1
$$


Demostración 6.2 Esta afirmación se puede demostrar mediante el teorema de la ganancia pequeña [9].

\section{SIMULACIÓN NUMÉRICA}

Para validar el observador del SOC propuesto, se ha realizado una simulación numérica. Los parámetros de la batería se resumen en la Tabla 1. Tomando estos parámetros, los cuales en una aplicación real son fáciles de medir o obtener su valor, solo es necesario introducir la corriente y los caudales que actúan como entradas.

Respecto a la corriente, se ha seleccionado un proceso de carga con un valor constante de $1.8 \mathrm{~A}$. Para el caso de los caudales $q_{1}$ y $q_{2}$, se ha considerado diferente, teniendo en cuenta que en la práctica nunca serán exactamente iguales. Se ha considerado un caudal de $100 \mathrm{ml} / \mathrm{min}$ en la parte del catolito y de $110 \mathrm{ml} / \mathrm{min}$ en el anolito.

\begin{tabular}{|c|c|}
\hline Variable & Valor [unidad] \\
\hline$v_{c}$ & $1[\mathrm{ml}]$ \\
\hline$v_{t}$ & $45[\mathrm{ml}]$ \\
\hline$c_{v_{-}}$ & $0.35\left[\mathrm{~mol} \cdot \mathrm{l}^{-1}\right]$ \\
\hline$c_{v_{+}}$ & $0.4\left[\mathrm{~mol} \cdot \mathrm{l}^{-1}\right]$ \\
\hline$c_{H+}(0)$ & $1.6\left[\mathrm{~mol} \cdot \mathrm{l}^{-1}\right]$ \\
\hline$E^{\theta}$ & $1.267[V]$ \\
\hline$r$ & $1.33[\Omega]$ \\
\hline$R$ & $8.311\left[\mathrm{~J} \cdot \mathrm{mol}^{-1} \cdot \mathrm{K}^{-1}\right.$ \\
\hline$T$ & $297[K]$ \\
\hline$F$ & $96.485\left[s \cdot A \cdot \mathrm{mol}^{-1}\right]$ \\
\hline
\end{tabular}

Además, la estimación de concentraciones se ha inicializado en un punto factible considerando que la batería está prácticamente descargada, presentando un error relativo inicial del $50 \%$. Como se supone que los coeficientes de difusión son desconocidos, sus valores estimados iniciales se han establecido en cero. En el sistema real, los valores se han fijado en $k_{2}=4.5 \cdot 10^{-11}$ y $k_{5}=1.65 \cdot 10^{-11}$.

En cuanto al ajuste del parámetro del observador, se han seleccionado los valores de $\alpha$ haciendo Hurwitz el polinomio presentado en (8). Para el caso de la constante $\epsilon$, se ha diseñado para garantizar un equilibrio entre el rechazo de perturbaciones y la sensibilidad al ruido. Por otro lado, para el estimador de coeficientes de difusión, el parámetro $\gamma$ se ha ajustado para presentar una tasa de convergencia de parámetros adecuada mientras se conserva la condición (19). Todos los valores del diseño del observador se resumen en la Tabla 2.

Para analizar la robustez del diseño en relación con el ruido del sensor, se ha realizado una simu-
Tabla 2: Parámetros del observador

\begin{tabular}{cc}
\hline Variable & Value \\
\hline$\alpha_{1}$ & $4 \cdot 10^{-4}$ \\
$\alpha_{2}$ & $5.81 \cdot 10^{-8}$ \\
$\alpha_{3}$ & $3.59 \cdot 10^{-12}$ \\
$\alpha_{4}$ & $7.8 \cdot 10^{-17}$ \\
$\epsilon$ & 0.5 \\
$\gamma$ & $1 \cdot 10^{-7}$ \\
\hline
\end{tabular}

lación de 800 segundos introduciendo un ruido de alta frecuencia de variación 0,001 con respecto a la medida de voltaje, que es un valor razonable para un sensor de voltaje.

Las simulaciones obtenidas muestran el correcto funcionamiento del acoplamiento entre el observador de estado y el observador de estimación de parámetros, obteniendo una estimación correcta tanto del SOC como de los parámetros de difusión. Para el caso del ECD, en la Figura 2 se muestra cómo se reduce el error de un $43 \%$ presentado en la inicialización hasta un $0.0082 \%$ visualizado al final de la simulación.

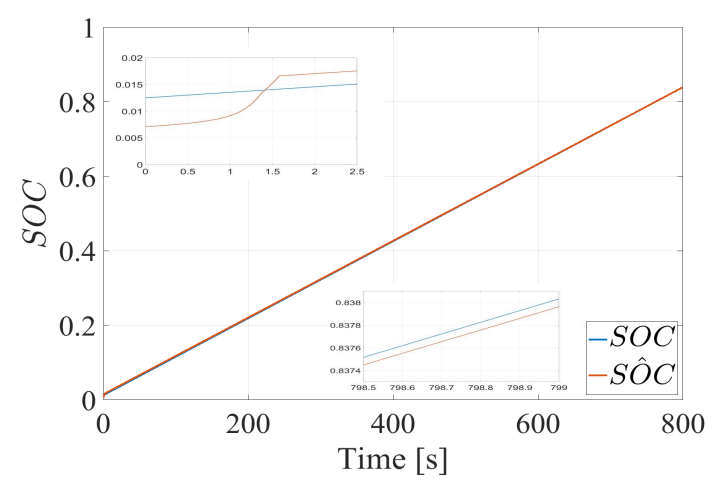

Figura 2: Estimación del $S O C$ (rojo) y su valor real (azul).

Se puede ver cómo el SOC aumenta al tratarse de un proceso de carga. Además, la evolución de las concentraciones de especies $x_{1}$ y $x_{2}$ presentan un comportamiento similar como se puede observar en la Figura 3.

Para los resultados del coeficiente de difusión, la Figura 4 muestra la estimación de los coeficientes $k_{2}$ y $k_{5}$, obteniendo que están correctamente ajustados a los reales. Para el caso de la estimación $k_{2}$, el error presentado es de $0,13 \%$, mientras que para la estimación de $k_{5}$ su valor no supera el $0,5 \%$. En detalle, se puede observar cómo la estimación $k_{5}$ presenta un sesgo. Este sesgo se debe a la introducción de ruido en la salida de voltaje, lo que impide la convergencia exacta del parámetro. 

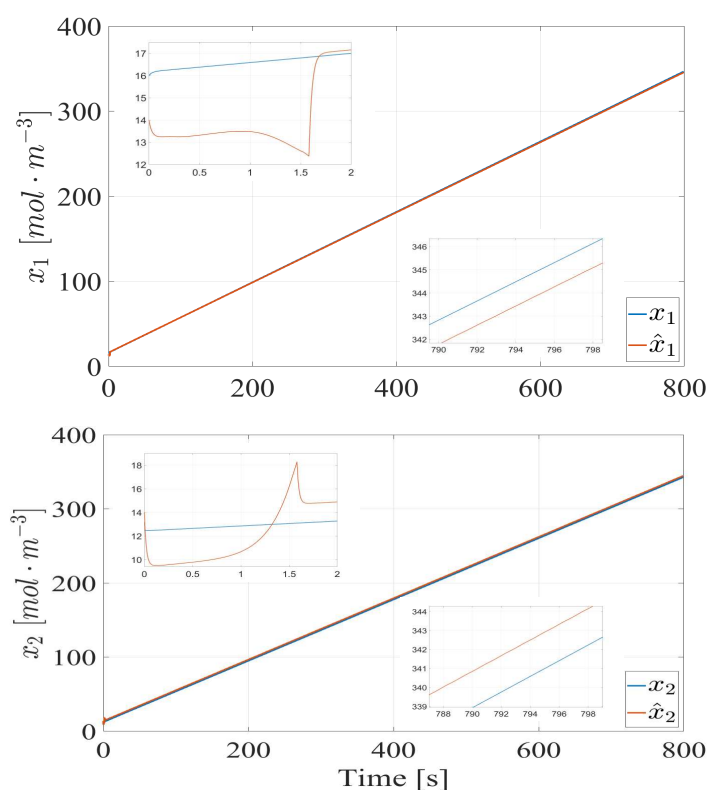

Figura 3: Estimación de estados (rojos) y valores reales (azul).

\section{CONCLUSIONES}

Este trabajo ha presentado un observador no lineal para estimar correctamente los estados de un VRFB suponiendo diferencias, en términos de caudales y especies de vanadio total, entre el catolito y el anolito. Por esa razón, se ha podido estimar por separado los dos SOC presentados en el sistema. Además, se ha realizado una estimación en línea de los parámetros de difusión utilizando un observador de alta ganancia $I \& I$. Los resultados se han validado en una simulación numérica en la que se considera un ruido de sensor significativo.

\section{Agradecimientos}

Este trabajo ha sido financiado por el CSIC bajo el proyecto PTI FLOWBAT 2021 (ref.642 201980E101), el Ministerio de Economía, Industria y Competitividad bajo el Proyecto DOVELAR (ref.RTI2018-096001-B-C32), y bajo la Agencia Estatatal de Investigación mediante la unidad de María de Maeztu Seal of Excellence to IRI (MDM-2016-0656).

\section{English summary}

\section{Estimation of the state of charge and diffusion coefficients in redox flow batteries}

\author{
Abstract \\ This paper presents an observer for the estima- \\ tion of the state of charge of redox flow batter-
}

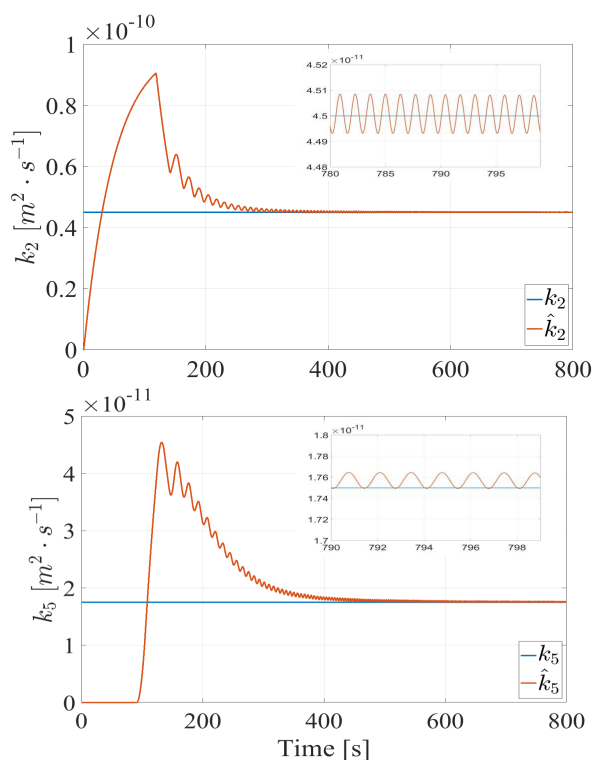

Figura 4: Estimación de los coeficientes de difusión.

ies. The observer is high gain and is based on a non-linear dynamic model, which uses a realistic approach differentiating between the total electrolyte concentrations in the two parts of the system. It is shown that the state of charge can be estimated by measuring cell voltage and a high gain observer. However, the precision of the estimate is affected by the uncertainty in the diffusion coefficients of the system. For this reason, the observer is strengthened by estimating adaptive parameters of immersion and invariance. Finally, the results are validated in a numerical simulation.

Keywords: Redox flow battery, state of charge, non-linear adaptive observer.

\section{Referencias}

[1] Astolfi, A., Ortega, R.: Immersion and invariance: a new tool for stabilization and adaptive control of nonlinear systems. IEEE Transactions on Automatic Control 48(4), 590-606 (2003), doi : 10.1109/TAC. 2003.809820

[2] Astolfi, D., Praly, L.: Output feedback stabilization for siso nonlinear systems with an observer in the original coordinates. In: 52nd IEEE Conference on Decision and Control. pp. 5927-5932 (2013), doi:10.1109/CDC. 2013.6760824

[3] Bernard, P., Praly, L., Andrieu, V., Hammouri, H.: On the triangular canonical 
form for uniformly observable controlled systems. Automatica 85, 293 - 300 (2017), doi:10.1016/j.automatica.2017.07.034

[4] Besançon, G.: Nonlinear observers and applications, vol. 363. Springer (2007)

[5] Cecilia, A., Costa-Castelló, R.: Observador de alta ganancia con zona muerta ajustable para estimar la saturación de agua líquida en pilas de combustible tipo pem. Revista Iberoamericana de Automática e Informática industrial 17(2), 169-180 (2020), doi:10.4995/riai.2020.12689

[6] Cecilia, A., Serra, M., Costa-Castelló, R.: Pemfc state and parameter estimation through a high-gain based adaptive observer. 21st IFAC World Congress (07 2020)

[7] Clemente, A., Costa-Castelló, R.: Redox flow batteries: A literature review oriented to automatic control. Energies 13, 4514 (09 2020), doi : 10.3390/en13174514

[8] IEA: Renewables 2019paris. In: IEEE (2019), https://www.iea.org/reports/renewables $-2019$

[9] Jiang, Z.P., Teel, A.R., Praly, L.: Smallgain theorem for iss systems and applications. Mathematics of Control, Signals and Systems 7(2), 95-120 (1994)

[10] Khalil, H.K., Praly, L.: High-gain observers in nonlinear feedback control. International Journal of Robust and Nonlinear Control 24(6), 993-1015 (2014), doi:10.1002/rnc. 3051

[11] Knehr, K., Kumbur, E.: Open circuit voltage of vanadium redox flow batteries: Discrepancy between models and experiments. Electrochemistry Communications 13(4), 342 - 345 (2011), doi:10.1016/j.elecom.2011.01.020

[12] Skyllas-Kazacos, M., Kazacos, M.: State of charge monitoring methods for vanadium redox flow battery control. Journal of
Power Sources 196(20), 8822 - 8827 (2011), doi : 10.1016/j.jpowsour.2011.06.080

[13] Wei, Z., Tseng, K.J., Wai, N., Lim, T.M., Skyllas-Kazacos, M.: Adaptive estimation of state of charge and capacity with online identified battery model for vanadium redox flow battery. Journal of Power Sources 332, 389 - 398 (2016), doi:10.1016/j.jpowsour.2016.09.123

[14] Wei, Z., Xiong, R., Lim, T.M., Meng, S., Skyllas-Kazacos, M.: Online monitoring of state of charge and capacity loss for vanadium redox flow battery based on autoregressive exogenous modeling. Journal of Power Sources 402, 252 - 262 (2018), doi:10.1016/j.jpowsour.2018.09.028

[15] Xiong, B., Zhang, H., Deng, X., Tang, J.: State of charge estimation based on sliding mode observer for vanadium redox flow battery. In: 2017 IEEE Power Energy Society General Meeting. pp. 1-5 (2017), doi : 10.1109/PESGM . 2017.8274042

[16] Xiong, B., Zhao, J., Wei, Z., SkyllasKazacos, M.: Extended kalman filter method for state of charge estimation of vanadium redox flow battery using thermal-dependent electrical model. Journal of Power Sources 262, 50 - 61 (2014), doi : 10.1016/j.jpowsour. 2014.03.110

[17] Young Man Cho, Rajamani, R.: A systematic approach to adaptive observer synthesis for nonlinear systems. IEEE Transactions on Automatic Control 42(4), 534-537 (1997), doi:10.1109/9.566664

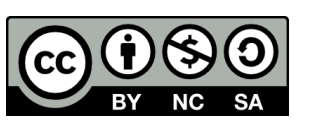

C) 2021 by the authors. Submitted for possible open access publication under the terms and conditions of the Creative Commons Attribution CC BY-NC-SA 4.0 license (https://creativecommons.org/licenses/by-ncsa/4.0/deed.es). 